completion of headache diaries. Simultaneous elimination of all known triggers is not generally recommended for nutritional reasons. A well-balanced diet is important, and skipping of meals or fasting should be avoided.

\title{
CRANIAL AUTONOMIC SYMPTOMS IN MIGRAINE
}

Cranial autonomic symptoms (CAS) in patients with migraine and cluster headaches $(\mathrm{CH})$ were characterized and compared in a prospective study of consecutive patients attending a headache clinic at Taipei Veterans General Hospital, Taiwan. CAS items surveyed were conjunctival injection, lacrimation, nasal congestion, rhinorrhoea, eyelid edema, and forehead/facial sweating. Of a total of 884 patients, 786 (625 women/161 men, mean age 40.1 (12.9) years) had migraine and 98 patients ( 11 women/87 men, mean age 36.2 $(10.5)$ years) had $\mathrm{CH}$. Migraine diagnoses were episodic without aura in $48 \%$, with aura in $5 \%$, chronic in $39 \%$, and probable migraine in $8 \%$. In the $\mathrm{CH}$ group, $99 \%$ had episodic $\mathrm{CH}$ and $1 \%$ had chronic $\mathrm{CH}$, a typical low incidence of chronic cases among Asians.

CAS occurred in $56 \%$ patients with migraine, and the incidence was similar in all migraine subtypes. Forehead/facial sweating in $28 \%$ of migraine patients was the commonest CAS, followed by lacrimation in $24 \%$. Migraine patients with CAS compared to those without had higher frequencies of severe migraine, nausea, photophobia and phonophobia, and vomiting. Patients with $\mathrm{CH}$ had a higher frequency of CAS than migraine patients. To differentiate migraine with $\mathrm{CAS}$ from $\mathrm{CH}$, the characteristic most predictive of migraine was bilateral CAS with either 1) mild to moderate intensity or 2) CAS occurring without headache. Lacrimation was the CAS with highest positive predictive value, specificity, and second highest sensitivity. (Lai T-H, Fuh J-L, Wang S-J. Cranial autonomic symptoms in migraine: characteristics and comparison with cluster headache. J Neurol Neurosurg Psychiatry Oct 2009;80(10):1116-1119). (Respond: Dr S-J Wang, Neurological Institute, Taipei Veterans General Hospital, Taipei, Taiwan 11217. E-mail: sjwang@vghtpe.gov.tw).

COMMENT. More than $50 \%$ of adults with migraine have cranial autonomic symptoms (CAS). Patients with CAS have more severe migraine often associated with photophobia, nausea and vomiting. Compared to those with cluster headaches, CAS with migraine are usually bilateral rather than unilateral and less severe.

Prof PJ Goadsby, San Francisco, in an editorial commentary, discusses the anatomy and physiology of CAS (J Neurol Neurosurg Psychiatry Oct 2009;80:1057-1058). The trigeminal-autonomic reflex is the basis for the symptoms. The effect is largely lateralized but innervation is also crossed. The pathway can be activated from the brain via connections from hypothalamus to superior salivatory nucleus. Comparing trigeminal autonomic cephalgias (TACS) and migraine, TACs are shorter in duration, sometimes seconds as in SUNCT/SUNA, minutes in paroxysmal hemicrania, and a few hours in cluster headache. In the clinical distinction of cluster headache and migraine, findings pointing to migraine include bilateral pain, attacks longer than 3 hours ( $>1-2$ hours in children), bilateral CAS, bilateral photophobia and phonophobia. Whereas patients with migraine are generally quiet, cluster headache patients are restless. Hemicrania continua response to indomethacin is another differentiating factor. CAS only at the time of headache should help in the distinction from sinus infection. In adults, migraine is more common in women, cluster headache in men. 
Cluster headache is uncommon in childhood. Onset is usually in the second and third decade. A retrospective review of cases attending a pediatric neurology clinic in Bristol, UK, between 2000 and 2005 identified 11 patients ( 7 male, 4 female) with median age of onset of 8.5 years (range 2-14). Median age at diagnosis was 11.5 years (range 7-17). Eight had episodic and 3 had chronic cluster headache. Most had cranial autonomic activation and agitated movement. (Majumdar A, Ahmed MA, Benton S. Eur J Paediatr Neurol 2008; Dec 22. (Epub ahead of print).

Maytal $\mathrm{J}$ et al modifications of the IHS criteria for pediatric migraine found that decreasing the length of attacks below 2 to 48 hours would increase the sensitivity of diagnosis, but adding associated autonomic symptoms of facial redness or pallor, while improving sensitivity, also decreased the specificity. The addition of CAS while helpful was not recommended. (Neurology 1997;48:602-607). Perhaps more attention to autonomic symptoms and behavior in diagnosis of children with migraine would be warranted.

\section{TOPIRAMATE-INDUCED COUGH IN MIGRAINE PROPHYLAXIS}

Three adults who developed intractable cough during topiramate prophylaxis of migraine are reported from the University of Padua and other centers in Italy. Cough developed early during the titration phase at dose levels of 75-100 mg/day, and resolved rapidly after withdrawal. Secondary causes of cough, including GERD, were excluded. The cough was episodic, dry, and very annoying, especially at night. Despite effective prevention of headache with topiramate, treatment was discontinued. Literature review revealed no previous case reports of cough as a side effect of topiramate treatment for migraine. (Maggioni F, Mampreso E, Mainardi F, Lisotto C, Malvindi ML, Zanchin G. Topiramateinduced intractable cough during migraine prophylaxis. Headache Oct 2009; on line). (Respond: Dr F Maggioni, Dept Neurosciences, University of Padua, Via Giustiniani 5, Padova, 35128, Italy).

COMMENT. Topiramate is a first-line treatment for migraine prophylaxis in adults. Adverse events in 20-25\% of patients may require discontinuation of treatment but are rarely severe. They include weight loss, dizziness, somnolence, paresthesias, impaired concentration and memory, and language difficulties. Cough has not been reported and the mechanism is unexplained. No patient received ACE inhibitors for hypertension, a known cause of dry cough in adults. Pubmed search for cough with topiramate treatment of childhood epilepsy or migraine found no reports.

\section{NEUROBEHAVIORAL DISORDERS}

\section{ISOLATED EPILEPTIFORM EEG DISCHARGES AND AUTISM}

The relationship between EEG abnormalities and neuropsychiatric disorders, and their possible clinical significance are reviewed by an investigator at Wayne State University, Detroit, MI, with special attention to the EEG and autism. Approximately one third of children with autistic spectrum disorder (ASD) develop epilepsy. Of 46 consecutive children

with autism (34 boys, and 12 girls, mean age $7.8+/-2.7$ years), 35\% had epilepsy (Canitano 